\title{
High-Harmonic Homodyne Detection of the Ultrafast Dissociation of $\mathrm{Br}_{2}$ Molecules
}

\author{
H. J. Wörner, J. B. Bertrand, P. B. Corkum, and D. M. Villeneuve \\ Joint Laboratory for Attosecond Science, National Research Council of Canada and University of Ottawa, \\ 100 Sussex Drive, Ottawa, Ontario, Canada KlA OR6
}

(Received 29 April 2010; published 1 September 2010)

\begin{abstract}
We report the time-resolved observation of the photodissociation of $\mathrm{Br}_{2}$ using high-harmonic generation (HHG) as a probe. The simultaneous measurement of the high-harmonic and ion yields shows that high harmonics generated by the electronically excited state interfere with harmonics generated by the ground state. The resulting homodyne effect provides a high sensitivity to the excited state dynamics. We present a simple theoretical model that accounts for the main observations. Our experiment paves the way towards the dynamic imaging of molecules using HHG.
\end{abstract}

Simultaneous imaging of the geometric and electronic structure of a molecule as it undergoes a chemical reaction is one of the main goals of modern ultrafast science. Techniques based on diffraction [1,2] measure the position of the atoms within the molecule with high accuracy but are much less sensitive to the electronic structure of the molecule, particularly the valence shell in which the chemical transformations originate. A new complementary approach exploits the rescattering of an electron removed from the molecule by a strong laser field to measure the structure of the molecule [3]. The associated recollision also leads to high-harmonic generation (HHG) that encodes the structure of the orbital to which the electron recombines $[4,5]$. So far, these methods have been applied only to the electronic ground state of molecules [6-9]. Ultrafast dynamics in molecules occur predominantly in excited electronic states which can only be prepared in small fractions when multiphoton processes need to be avoided.

We extend high-harmonic spectroscopy from probing static molecular structure to probing photochemical dynamics. Using the impulsive photodissociation of molecular bromine, we show that the electromagnetic interference between high harmonics generated from the molecular ground state and the excited state occurs on the attosecond time scale. The coherent addition of the emitted radiation results in high visibility of the excited state dynamics despite the low excited state fraction.

The photorecombination step in high-harmonic generation is essentially a time reversal of photoionization. Before describing the experiment, it is useful to compare and contrast the two approaches. In femtosecond photoelectron spectroscopy, single-photon absorption creates a photoelectron which encodes information of the molecule's electronic structure in its spectrum $[10,11]$. In femtosecond high-harmonic spectroscopy, similar detailed information [12,13] is carried in the emitted photon. In photoelectron spectroscopy, different initial (neutral) and final (ionic) electronic states are distinguished through the photoelectron energy. In high-harmonic spectroscopy, the broadband recolliding electron wave can only recombine to vacant states, which are selectively created by the tunnel ionization step. Tunnel ionization is exponentially sensitive to the binding energy of each ionization channel. The simultaneous measurement of a broad photon spectrum and the selection of the probed state by tunneling both point to greater simplicity for high-harmonic spectroscopy. However, the emitted photons originating from different initial electronic states overlap spectrally, adding an undesirable complexity to the interpretation of highharmonic spectroscopy. We show that this apparent complexity becomes an advantage as the unexcited molecules can serve as a local oscillator against which we measure the excited state dynamics. Just as in a radio receiver, the local oscillator makes a weak signal that would be otherwise difficult to observe, readily visible. Moreover, the coherent detection provides a high sensitivity to the phase of the radiation, which reflects the evolution of the ionization potential along the dissociation coordinate.

The experimental setup consists of a chirped-pulse amplified titanium-sapphire femtosecond laser system, a highharmonic source chamber equipped with a pulsed valve, and an xuv spectrometer. The laser system provides $800 \mathrm{~nm}$ pulses of $32 \mathrm{fs}$ duration (FWHM). The laser beam is split into two parts of variable intensities using a half-wave plate and a polarizer. The minor part of the energy is sent through a 2:1 telescope and used to generate $400 \mathrm{~nm}$ radiation in a type I BBO of $60 \mu \mathrm{m}$ thickness. The major part is sent through a computer-controlled delay stage and is recombined with the $400 \mathrm{~nm}$ radiation using a dichroic beam splitter. The combined beams are focused into the chamber using a $f=50 \mathrm{~cm}$ spherical mirror.

High-order harmonics are generated in a supersonic expansion of $\mathrm{Br}_{2}$ seeded in 2 bars of helium. The helium carrier gas is sent through liquid $\mathrm{Br}_{2}$ kept at room temperature. Bromine molecules are excited by single-photon absorption at $400 \mathrm{~nm}$ to the repulsive $C^{1} \Pi_{1 u}$ state (see Fig. 1) and high harmonics are generated in the strong 


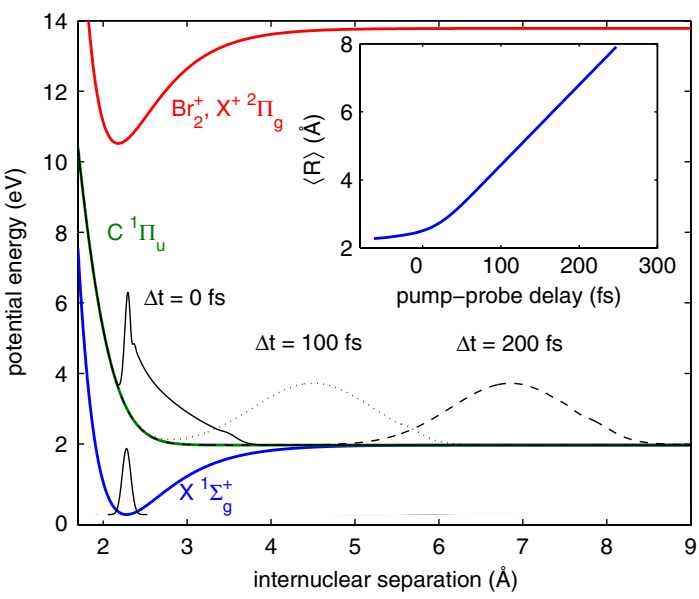

FIG. 1 (color online). Potential energy curves of $\mathrm{Br}_{2}\left(X^{1} \Sigma_{g}^{+}\right.$ ground state and $C^{1} \Pi_{1 u}$ excited state) and $\mathrm{Br}_{2}^{+}\left(X^{+2} \Pi_{g}\right.$ ground state). The shape of the nuclear wave packet in the excited state after selected delays $\Delta t$ is also shown. The wave packets were obtained by numerical propagation assuming a 40 fs excitation pulse centered at $400 \mathrm{~nm}$. The inset shows the expectation value of the internuclear separation as a function of the pump-probe delay.

$800 \mathrm{~nm}$ field. The focus of both beams is placed $\sim 1 \mathrm{~mm}$ before the pulsed molecular jet expanding through a nozzle of $250 \mu \mathrm{m}$ diameter. This setup minimizes the effect of phase mismatch and reabsorption of the high-harmonic radiation and leads to the observation of the singlemolecule response [14]. The typical pulse energies of $1.5 \mathrm{~mJ}(800 \mathrm{~nm})$ and $5 \mu \mathrm{J}(400 \mathrm{~nm})$ result in intensities of $1.5 \times 10^{14} \mathrm{~W} / \mathrm{cm}^{2}(800 \mathrm{~nm})$ and $5 \times 10^{11} \mathrm{~W} / \mathrm{cm}^{2}$. The total number of produced ions is measured by a wire mesh located $10 \mathrm{~cm}$ below the nozzle orifice. We have verified that the response of the ion detector was linear. The high harmonics are spectrally resolved using an aberrationcorrected xuv grating and imaged by a microchannel plate detector backed with a phosphor screen using a chargecoupled device camera. The harmonic intensities are extracted by integrating the images spatially and spectrally.

Figure 1 shows the relevant potential energy curves of $\mathrm{Br}_{2}$ and $\mathrm{Br}_{2}^{+}$. Single-photon excitation at $400 \mathrm{~nm}$ from the $X^{1} \Sigma_{g}^{+}$ground state leads almost exclusively to the repulsive $C^{1} \Pi_{1 u}$ state which dissociates into two bromine atoms in their ground spin-orbit state $\left({ }^{2} P_{3 / 2}, m_{J}=1 / 2\right)$ [15]. The figure also shows the shape of the vibrational wave function in the ground state and the calculated nuclear wave packet on the excited state surface at selected delays $\Delta t$ after excitation by a $40 \mathrm{fs}$ pump pulse centered at $400 \mathrm{~nm}$. The ${ }^{2} \Pi_{3 / 2 g}$ ground state curve of $\mathrm{Br}_{2}^{+}$is also shown to illustrate the variation of the ionization potential with the internuclear distance.

Figure 2 shows the observed harmonic and ion signals in a pump-probe experiment with perpendicular polarizations. The power of H19 decreases during the excitation, reaches a minimum after the peak of the $400 \mathrm{~nm}$ pulse, and then recovers to its initial power level. In contrast, the ion yield increases, reaching its maximum after the peak of the

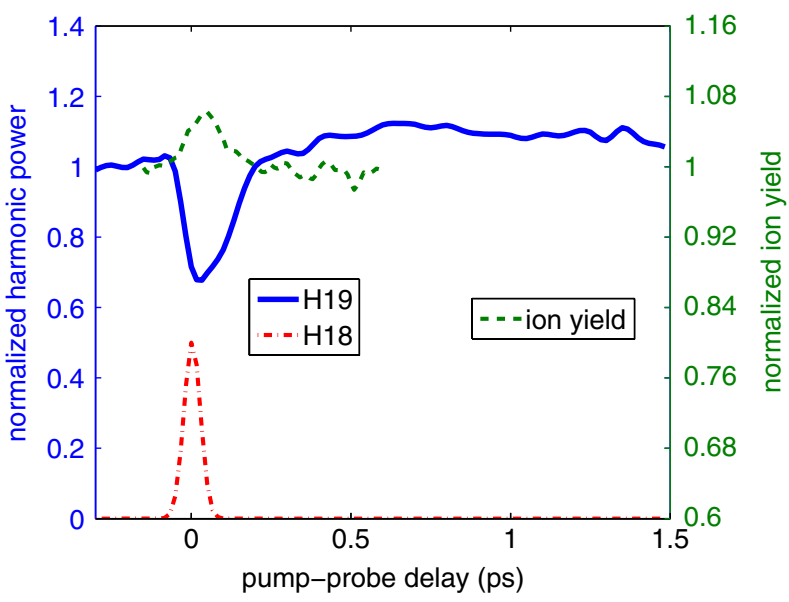

FIG. 2 (color online). Power of harmonics 19 (full line) and 18 (dash-dotted line) as a function of the delay between a $400 \mathrm{~nm}$ pump pulse and a perpendicularly polarized $800 \mathrm{~nm}$ pulse generating high harmonics (left-hand axis). The temporal overlap of 400 and $800 \mathrm{~nm}$ pulses leads to the emission of even-order harmonics, like H18. The total ion yield (dashed line, right-hand axis) was measured under identical conditions but with higher statistics.

$400 \mathrm{~nm}$ pulse and subsequently decreases to its initial level. The maximum increase in ion yield amounts to $7 \%$, whereas the harmonic signal is depleted by up to $30 \%$. The signal of H19 and the ion yield have been normalized to unity at negative delays. This signal level corresponds to all molecules being in the ground electronic state. Temporal overlap of the 800 and $400 \mathrm{~nm}$ pulses leads to the appearance of even-order harmonics [16] which provide the time origin and a high-order cross correlation ( 50-60 fs).

When $\mathrm{Br}_{2}$ is excited to the $C^{1} \Pi_{1 u}$ state, the ionization potential for the removal of the most weakly bound electron is reduced from 10.5 to $7.5 \mathrm{eV}$, explaining the observed increase in the ion yield. The rising part of the ion yield curve reflects the buildup of the excited state population during the excitation pulse. $\mathrm{As}_{\mathrm{Br}_{2}}$ dissociates along the repulsive $C{ }^{1} \Pi_{1 u}$ state, the ionization potential increases from 7.5 to $11.8 \mathrm{eV}$, resulting in a decreasing ionization rate of the excited state. Since the ionization rate increases at early delays, one might expect that the harmonic yield would also increase. However, the opposite is observed. Moreover, the variation of the harmonic signal is much larger than that of the ion signal and exceeds the excitation fraction by a factor of 2 . These results clearly demonstrate a destructive interference between harmonics emitted by the excited molecules and those emitted by the ground state molecules. Destructive interference is the origin of the opposite behavior of ion and harmonic yield. To better understand the origin of the interference, we now compare the temporal evolution of different harmonic orders.

Figure 3 shows the evolution of the power of a range of odd harmonics (H13-23, $\lambda=61-35 \mathrm{~nm}$ ) with the pumpprobe delay in an experiment using parallel polarizations. 
The low-order odd harmonics (H13-19) approximately recover the power they had prior to excitation within a time scale of $300 \mathrm{fs}$ whereas the high-order harmonics (H21-23) remain at a lower power level. The observed behavior in parallel and perpendicular (not shown) polarizations is qualitatively similar, although the time scales differ slightly.

The variation of the observed modulation with harmonic order demonstrates that the interference is tied to the nature of the trajectory of the continuum electron. The temporal extension of the short electron trajectories spans a range of $0-1.7$ fs [17]. The electron transit time of harmonics emitted by species of different ionization potentials thus differs by hundreds of attoseconds [18], which is the time scale on which the interference occurs. In what follows, we develop a theoretical model to calculate the phase of the highharmonic radiation emitted by the dynamically evolving excited state molecules.

We treat the problem within the Born-Oppenheimer approximation and write the molecular wave function at a delay $\Delta t$ after excitation as

$$
\Psi(\vec{r}, R, \Delta t)=c_{g} \Phi_{g}(\vec{r}) \chi_{g}(R)+c_{e} \Phi_{e}(\vec{r}, R) \chi_{e}(R),
$$

where $\vec{r}$ is a set of electronic coordinates and $R$ is the internuclear separation. $\Phi_{g}(\vec{r})$ and $\Phi_{e}(\vec{r}, R)$ are the electronic wave functions of the ground and excited states and $\chi_{g}$ and $\chi_{e}$ are the corresponding normalized nuclear wave functions. The radiated electric field corresponding to the photon energy $\Omega$ can be written as [19]

$$
\begin{aligned}
E(\Omega) \propto & \left|c_{g}\right|^{2}\left\langle\chi_{g} \mid \chi_{g}\right\rangle E_{g}(\Omega)+\left|c_{e}\right|^{2}\left\langle\chi_{e} \mid \chi_{e}\right\rangle E_{e}(\Omega) \\
& +c_{e}^{*} c_{g}\left\langle\chi_{e} \mid \chi_{g}\right\rangle E_{e g}(\Omega)+c_{g}^{*} c_{e}\left\langle\chi_{g} \mid \chi_{e}\right\rangle E_{g e}(\Omega),
\end{aligned}
$$

where $E_{i(j)}$ are complex spectral components. The first term in Eq. (2), proportional to $\left|c_{g}\right|^{2}$, corresponds to harmonic emission from the ground state of the molecule

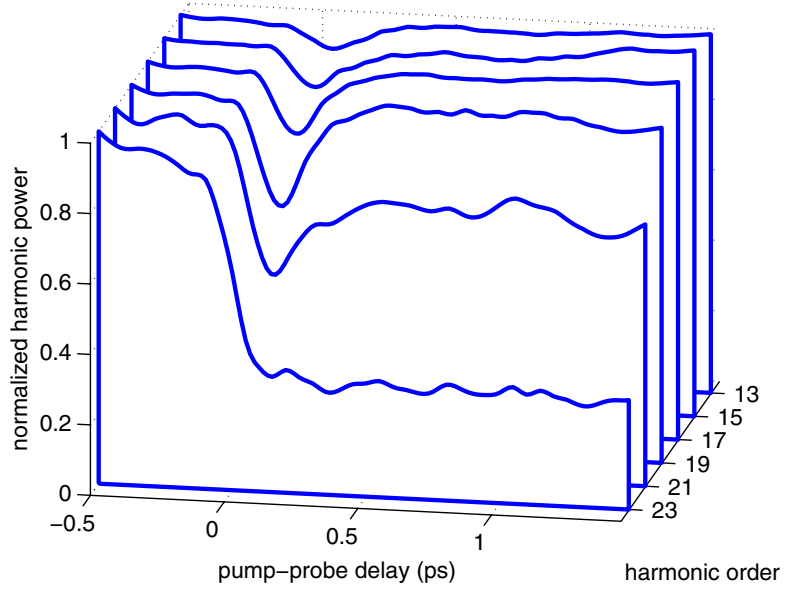

FIG. 3 (color online). Observed variation of the power of harmonics 13 to 23 as a function of the time delay between a $400 \mathrm{~nm}$ pump pulse and a parallel polarized $800 \mathrm{~nm}$ pulse generating high harmonics. The intensities have been normalized to their respective values at negative time delays. alone, the second, proportional to $\left|c_{e}\right|^{2}$, represents emission from the excited state. Radiation can also be emitted by ionization of either of the two electronic states and recombination to the other one [20]. These cross terms are proportional to the overlap of the nuclear wave functions $\left\langle\chi_{e} \mid \chi_{g}\right\rangle$, which in our case decays to zero within $40 \mathrm{fs}$ of the excitation. Therefore, we will only consider the first two terms. The total radiated field at photon energy $\Omega=q \omega$ can thus be represented as $E_{q}=(1-r) d_{g} e^{i \phi_{g}}+$ $r d_{e} e^{i \phi_{e}}$, where $d_{g}$ and $d_{e}$ are the real $q$ th harmonic amplitudes and $\phi_{g}$ and $\phi_{e}$ are the $q$ th harmonic phases of the ground and excited states, respectively, and $r=\left|c_{e}\right|^{2}$ is the excited state fraction.

Within the strong-field approximation, the phase of harmonic $q$ is given by [21]

$$
\phi\left(q, t_{i}, t_{r}\right)=\frac{1}{2} \int_{t_{i}}^{t_{r}}\left(\vec{A}\left(t_{i}\right)-\vec{A}(t)\right)^{2} d t+I_{p}\left(t_{r}-t_{i}\right)-q \omega t_{r},
$$

where $t_{i}$ and $t_{r}$ are the moments of ionization and recombination, respectively, $\vec{A}(t)$ is the vector potential of the laser field, and $I_{p}$ is the ionization potential of the molecule. We use classical simulations of the short electron trajectories to calculate the total phase [17]. The first term in Eq. (3) is obtained by numerical integration over these trajectories. Additional contributions to the high-harmonic phase originate from the ionization and recombination steps but are neglected within the strong-field approximation.

The dissociation of $\mathrm{Br}_{2}$ in the $C^{1} \Pi_{1 u}$ state is simulated by propagating a wave packet on the repulsive surface using the split operator technique [22]. The potential energy curves of the $X^{1} \Sigma_{g}^{+}$ground state, the $C^{1} \Pi_{1 u}$ state, and the $X^{+}{ }^{2} \Pi_{g}$ ground state of the cation are shown in Fig. 1. We calculate the total harmonic power as a coherent sum of the emission from the ground and excited electronic states

$$
\left.\left.P \propto\left|(1-r) d_{g} e^{i \phi_{g}}+r \int d R\right| \chi_{e}(R)\right|^{2} d_{e}(R) e^{i \phi_{e}(R)}\right|^{2} .
$$

In the calculation, the excited state fraction is $r=0.14$, matching the experimental conditions. We use an $R$-independent photorecombination amplitude but account for the increase in the number of emitters as one molecule dissociates into two atoms by modeling $d_{e}(R)=$ $(\operatorname{erf}(R / \AA-4)+1) / 2+1$, i.e., a smoothed step function increasing from 1 to 2 . $\phi_{e}$ depends on $R$ through the coordinate dependence of the ionization potential and is calculated according to Eq. (3).

The comparison of Figs. 3 and 4 shows that the model provides a qualitative explanation of the experimental results. The power of all harmonics decreases during the excitation pulse and reaches a minimum after the peak of the excitation pulse. The power of the low harmonics (H13-H19) is found to partially recover, in agreement with the experiment. The power of the high harmonics remains low when the dissociation dynamics is over. This is the consequence of a destructive interference 


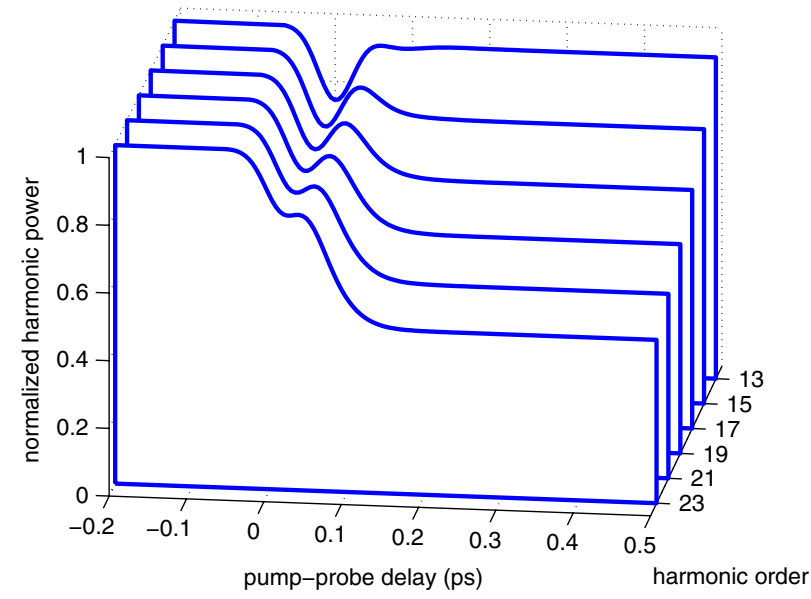

FIG. 4 (color online). Calculated variation of the power of harmonics 13 to 23 using Eq. (4). The calculations rely on a wave packet obtained by numerical propagation on the repulsive $C$ state as shown in Fig. 1 and detailed in the text.

between the harmonics emitted by $\mathrm{Br}_{2}$ molecules and $\mathrm{Br}$ atoms. The amplitude at long time delays relative to the amplitude at negative delays decreases with increasing harmonic order. This feature is observed in the experiment and is also reproduced in the calculations. The calculated phase difference of the harmonic emission between $\mathrm{Br}_{2}$ molecules and $\mathrm{Br}$ atoms indeed increases from $1.6 \mathrm{rad}$ for $\mathrm{H} 13$ to $2.5 \mathrm{rad}$ for $\mathrm{H} 23$ under our conditions, explaining the increasingly destructive interference. The overall agreement between theory and experiment at early and late times indicates that the proposed interference model properly describes the physical phenomenon.

A noticeable difference between the experiment and the simulation is the time scale on which the high-harmonic signal reaches its asymptotic level. In the simulation, the time scale is close to $100 \mathrm{fs}$ which corresponds to the time it takes to double the internuclear separation. In the experiment, the signal takes about $300 \mathrm{fs}$ to recover. This time scale is surprisingly long and indicates that the transition between the molecular and atomic character of HHG takes significantly longer than what might be expected. The comparison between theory and experiment thus allows us to anticipate that the photorecombination amplitude varies with internuclear separation and that it only reaches the atomic value at very large internuclear separations ( $>9 \AA$ ). More subtle differences between the experiment and the model calculations may also arise from contributions of the photorecombination phase to the harmonic phase and from the contribution of multiple orbitals to HHG. The full characterization of high-harmonic emission from dynamically evolving systems will thus require a simultaneous measurement of amplitude and phase of the high harmonics [23].

In conclusion, we have demonstrated that interference occurs on the attosecond time scale between high harmonics emitted by the excited state of a dissociating molecule and those emitted by the ground state. This interference occurs in a single molecule but it is electromagnetic in nature. We have shown that the intrinsic homodyne nature of the method permits a very sensitive detection of chemical changes occurring on the femtosecond time scale and allows us to follow the breaking of a chemical bond. This opens up the possibility of tomographic imaging of molecular dynamics [4]. The method will allow us to follow the evolution of valence orbitals during a chemical reaction. Moreover highharmonic generation offers the potential of attosecond temporal resolution. This advantage may be applied to study the electronic dynamics launched in the molecular cation by tunnel ionization and their dependence on the reaction coordinate.

H. J. W. acknowledges financial support from the Swiss National Science Foundation (SNF). We acknowledge funding through NSERC and AFOSR.

[1] R. Neutze, R. Wouts, D. van der Spoel, E. Weckert, and J. Hajdu, Nature (London) 406, 752 (2000).

[2] H. Ihee et al., Science 291, 458 (2001).

[3] M. Meckel et al., Science 320, 1478 (2008).

[4] J. Itatani et al., Nature (London) 432, 867 (2004).

[5] S. Baker et al., Phys. Rev. Lett. 101, 053901 (2008).

[6] T. Kanai, S. Minemoto, and H. Sakai, Nature (London) 435, 470 (2005).

[7] C. Vozzi et al., Phys. Rev. Lett. 95, 153902 (2005).

[8] N.L. Wagner et al., Proc. Natl. Acad. Sci. U.S.A. 103, 13279 (2006).

[9] W. Li et al., Science 322, 1207 (2008).

[10] L. Nugent-Glandorf et al., Phys. Rev. Lett. 87, 193002 (2001).

[11] P. Wernet et al., Phys. Rev. Lett. 103, 013001 (2009).

[12] H. J. Wörner, H. Niikura, J. B. Bertrand, P. B. Corkum, and D. M. Villeneuve, Phys. Rev. Lett. 102, 103901 (2009).

[13] A.-T. Le, R. R. Lucchese, S. Tonzani, T. Morishita, and C. D. Lin, Phys. Rev. A 80, 013401 (2009).

[14] A. D. Shiner et al., Phys. Rev. Lett. 103, 073902 (2009).

[15] T. P. Rakitzis and T. N. Kitsopoulos, J. Chem. Phys. 116, 9228 (2002).

[16] H. Eichmann et al., Phys. Rev. A 51, R3414 (1995).

[17] P. B. Corkum, Phys. Rev. Lett. 71, 1994 (1993).

[18] T. Kanai, E. J. Takahashi, Y. Nabekawa, and K. Midorikawa, Phys. Rev. Lett. 98, 153904 (2007).

[19] M. Spanner and P. Brumer, Phys. Rev. A 78, 033840 (2008).

[20] J. B. Watson, A. Sanpera, X. Chen, and K. Burnett, Phys. Rev. A 53, R1962 (1996).

[21] M. Lewenstein, P. Balcou, M. Y. Ivanov, A. L'Huillier, and P. B. Corkum, Phys. Rev. A 49, 2117 (1994).

[22] D. J. Tannor, Introduction to Quantum Mechanics: A TimeDependent Perspective (University Science Books, Sausalito, 2007).

[23] H. J. Wörner, J.B. Bertrand, D. V. Kartashov, P. B. Corkum, and D. M. Villeneuve, Nature (London) 466, 604 (2010). 